University of Wollongong

Research Online

Faculty of Engineering - Papers (Archive)

Faculty of Engineering and Information

Sciences

20-5-2005

\title{
The Powerful Outburst in Hercules A
}

P. Nulsen

University of Wollongong

D. C. Hambrick

Harvard-Smithsonian Center for Astrophysics, Cambridge, USA

B. R. McNamara

Ohio University, USA

D. Rafferty

Ohio University, USA

L. Birzan

Ohio University, USA

See next page for additional authors

Follow this and additional works at: https://ro.uow.edu.au/engpapers

Part of the Engineering Commons

https://ro.uow.edu.au/engpapers/295

\section{Recommended Citation}

Nulsen, P.; Hambrick, D. C.; McNamara, B. R.; Rafferty, D.; Birzan, L.; Wise, M. W.; and David, L. P.: The Powerful Outburst in Hercules A 2005.

https://ro.uow.edu.au/engpapers/295

Research Online is the open access institutional repository for the University of Wollongong. For further information contact the UOW Library: research-pubs@uow.edu.au 


\section{Authors}

P. Nulsen, D. C. Hambrick, B. R. McNamara, D. Rafferty, L. Birzan, M. W. Wise, and L. P. David 


\title{
THE POWERFUL OUTBURST IN HERCULES A
}

\author{
P. E. J. Nulsen, ${ }^{1,2}$ D. C. Hambrick, ${ }^{1,3}$ B. R. McNamara, ${ }^{4}$ D. Rafferty, ${ }^{4}$ L. Birzan, ${ }^{4}$ M. W. Wise, ${ }^{5}$ and L. P. David ${ }^{1}$ \\ Received 2004 December 1; accepted 2005 April 14; published 2005 April 28
}

\begin{abstract}
The radio source Hercules A resides at the center of a cooling flow cluster of galaxies at redshift $z=0.154$. A Chandra X-ray image reveals a shock front in the intracluster medium (ICM) surrounding the radio source, about $160 \mathrm{kpc}$ from the active galactic nucleus (AGN) that hosts it. The shock has a Mach number of 1.65, making it the strongest of the cluster-scale shocks driven by an AGN outburst found so far. The age of the outburst is $\simeq 5.9 \times 10^{7} \mathrm{yr}$, its energy $\sim 3 \times 10^{61} \mathrm{ergs}$, and its mean power $\sim 1.6 \times 10^{46} \mathrm{ergs} \mathrm{s}^{-1}$. As for the other large AGN outbursts in cooling flow clusters, this outburst overwhelms radiative losses from the ICM of the Hercules A Cluster by a factor of $\sim 100$. It adds to the case that AGN outbursts are a significant source of preheating for the ICM. Unless the mechanical efficiency of the AGN in Hercules A exceeds 10\%, the central black hole must have grown by more than $1.7 \times 10^{8} M_{\odot}$ to power this one outburst.
\end{abstract}

Subject headings: cooling flows - galaxies: clusters: individual (Hercules A) — intergalactic medium — X-rays: galaxies: clusters

\section{INTRODUCTION}

It is not yet clear which heating mechanism (e.g., Narayan \& Medvedev 2001; Motl et al. 2004) is chiefly responsible for preventing gas from cooling in cluster cooling flows (Peterson et al. 2003), but the most promising is heating by a central AGN (Tabor \& Binney 1993; Tucker \& David 1997). Heating and cooling rates are linked if the AGN is fed by cooled or cooling gas. Such feedback could maintain otherwise unstable cool cores, explaining the prevalence of cooling flows (Fabian 1994). AGN-powered radio-lobe cavities (e.g., Carilli et al. 1994; McNamara et al. 2000; Fabian et al. 2000) heat the ICM (Churazov et al. 2002), but not enough to make up for radiative losses (Birzan et al. 2004). Weak "cocoon" shocks, long expected in models of jet-fed radio lobes (e.g., Scheuer 1974; Heinz et al. 1998), have been found in a number of systems (Fabian et al. 2003; Forman et al. 2005; McNamara et al. 2005; Nulsen et al. 2005). They represent additional heating due to AGN outbursts, and they provide a new tool for determining ages and energies of AGN outbursts.

In this Letter, we report the discovery of a shock front generated by the AGN outburst that powers Hercules A. One of the brightest radio sources in the sky (Dreher \& Feigelson 1984; Gizani \& Leahy 2003), Hercules A resides at the center of a cluster of galaxies with X-ray luminosity $\simeq 5 \times 10^{44} \mathrm{ergs} \mathrm{s}^{-1}$, at redshift $z=0.154$ (Siebert et al. 1999; Gizani \& Leahy 2004). Despite its high radio luminosity, Hercules A lacks bright radio hot spots and so belongs to Fanaroff-Riley class I, but with an unusual, jet-dominated, radio morphology (Dreher \& Feigelson 1984; Gizani \& Leahy 2003). Using Einstein spectra, White et al. (1997) found a formal cooling rate of zero for the Hercules A Cluster, but the high central density we find $\left(n_{e} \gtrsim 0.1 \mathrm{~cm}^{-3}\right)$ gives it a central cooling time typical of a cooling flow cluster.

${ }^{1}$ Harvard-Smithsonian Center for Astrophysics, 60 Garden Street, Cambridge, MA 02138; pnulsen@cfa.harvard.edu.

${ }^{2}$ On leave from the University of Wollongong.

${ }^{3}$ Harvey Mudd College, 301 East Foothill Boulevard, Claremont, CA 91711.

${ }^{4}$ Astrophysical Institute, and Department of Physics and Astronomy, Ohio University, Clippinger Laboratories, Athens, $\mathrm{OH} 45701$.

${ }^{5}$ Center for Space Research, Building NE80-6015, Massachusetts Institute of Technology, Cambridge, MA 02139.
In $\S 2$ we give details of the observations and data reduction, and in $\S 3$ we discuss the main features of the Chandra image of Hercules A. Properties of the shock are discussed in $\S 4$, and its implications in $\S 5$. A flat $\Lambda \mathrm{CDM}$ cosmology, with $H_{0}=70 \mathrm{~km} \mathrm{~s}^{-1} \mathrm{Mpc}^{-1}$ and $\Omega_{m}=0.3$, is assumed throughout, giving a scale of $2.67 \mathrm{kpc} \operatorname{arcsec}^{-1}$ for Hercules A.

\section{OBSERVATIONS AND DATA REDUCTION}

Hercules A was observed with Chandra for $14.8 \mathrm{ks}$ on 2001 July 25, in VFAINT mode with ACIS-S at the aim point (ObsID 1625). For the analysis here, the event list was reprocessed using recent calibrations. It was screened to remove $A S C A$ grades 1, 5, and 7 and bad pixels. Periods of high particle background were removed following the method of M. Markevitch, ${ }^{6}$ leaving $12.4 \mathrm{ks}$ of good exposure time. After cleaning, the mean count rate in ACIS-S1 was 0.136 counts $\mathrm{s}^{-1}, \sim 3.5 \sigma$ $(9 \%)$ higher than expected, ${ }^{7}$ suggesting some residual contamination due to particle background. Data were processed to correct for the time dependence of the ACIS gain ${ }^{8}$ and filtered according to the prescription of A. Vikhlinin ${ }^{9}$ to reduce particle background. Background event files were created by processing standard ACIS background files in the same manner as the data. Point sources were identified manually for removal from spectra and surface brightness profiles. Ancillary response files and response matrix files for extended regions are weighted by number of events. Ancillary response files are corrected to allow for the reduction in low-energy response due to contaminant on the ACIS filters.

\section{THE X-RAY IMAGE OF HERCULES A}

The image in the upper panel of Figure 1 shows the filtered and calibrated events from the raw Chandra data (evt2 file) for Hercules A, binned by a factor of 4 . The lower panel shows a $0.3-7.5 \mathrm{keV}$ image made after the cleaning and reprocessing described above. The image has been smoothed with a 2 " Gaussian and divided by a $\beta$-model, with a $42^{\prime \prime}$ core radius and a

\footnotetext{
${ }^{6}$ See http://hea-www.harvard.edu/ maxim/axaf/acisbg.

${ }^{7}$ See http://hea-www.harvard.edu/ maxim/axaf/acisbg/data/README.

See http://hea-www.harvard.edu/ alexey/acis/tgain.

${ }^{9}$ See http://cxc.harvard.edu/cal/Acis/Cal_prods/vfbkgrnd.
} 

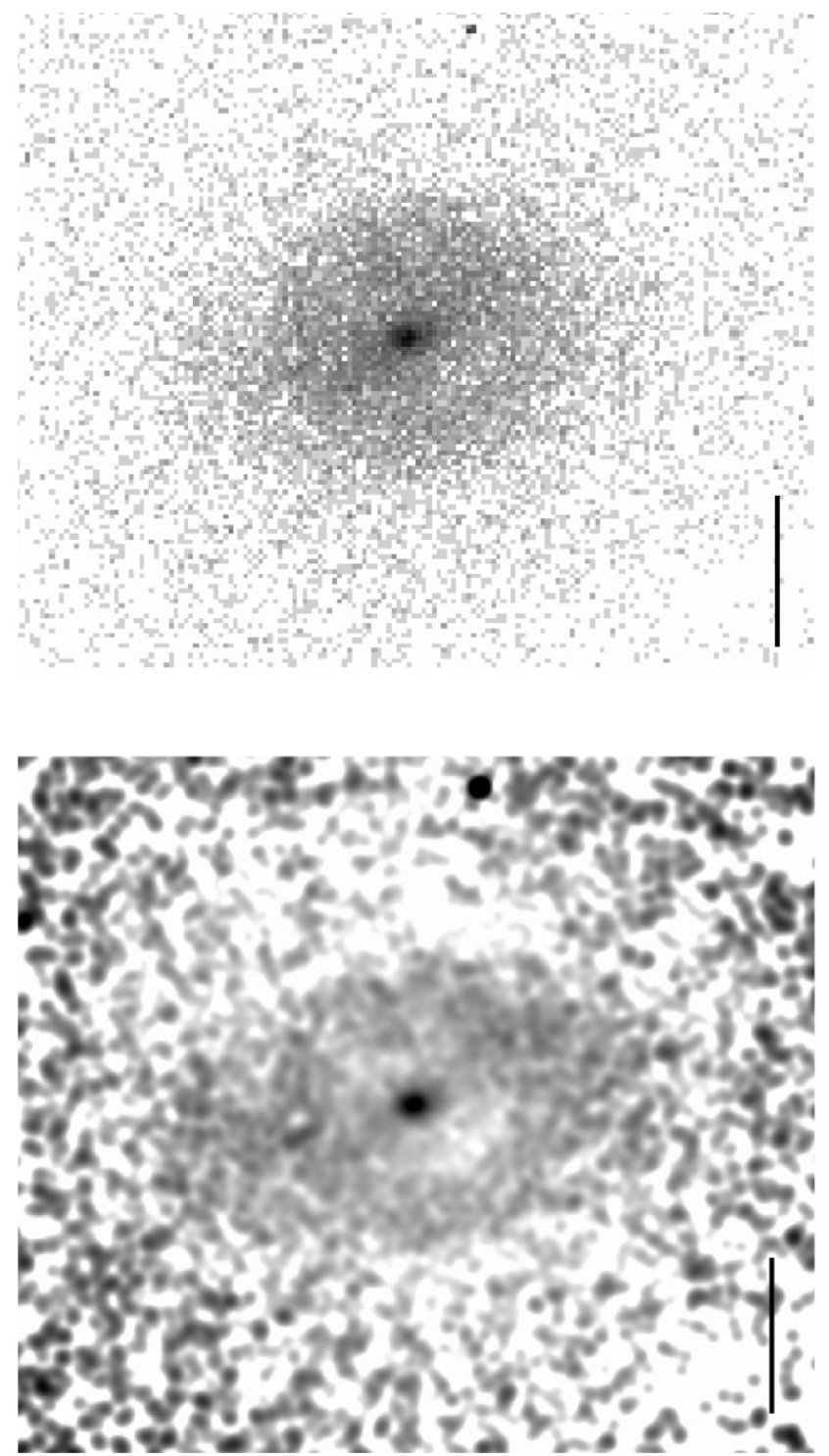

FIG. 1.-Upper panel: $0.3-7.5 \mathrm{keV}$ Chandra image of Hercules A made from the distributed evt 2 file binned by a factor of 4 . Lower panel: $0.3-7.5 \mathrm{keV}$ image of Hercules A made from the cleaned, reprocessed data, smoothed with a $2^{\prime \prime}$ Gaussian and divided by a $\beta$-model. The scale bar in each panel is $1^{\prime}(160 \mathrm{kpc})$ in length. The bright central region $\sim 1^{\prime}$ in radius is surrounded by the shock front. The southwest cavity is $\sim 0.5$ from the central peak.

$\beta$ of 0.6 , centered on the X-ray peak (core radius from Gizani $\&$ Leahy 2004 , but smaller $\beta$ ). Division by the $\beta$-model reduces the radial variation of surface brightness, making it easier to discern substructure over a substantial range of radius. Each image has a $1^{\prime}$ scale bar.

Although the central peak of the X-ray image is prominent, it is well resolved by Chandra, and there is no sign of a pointlike AGN (cf. Trussoni et al. 2001). A striking feature of the $\mathrm{X}$-ray image is the bright region, roughly $1^{\prime}$ in radius, that stands out in the upper panel of Figure 1. This has a similar size to the radio emission and extends to the east and west around the radio lobes (see Fig. 1, lower panel, and Fig. 2). Its shape and association with the radio source suggest that it is the shocked cocoon of the expanding radio lobes (Scheuer 1974; Heinz et al. 1998). The break in surface brightness that

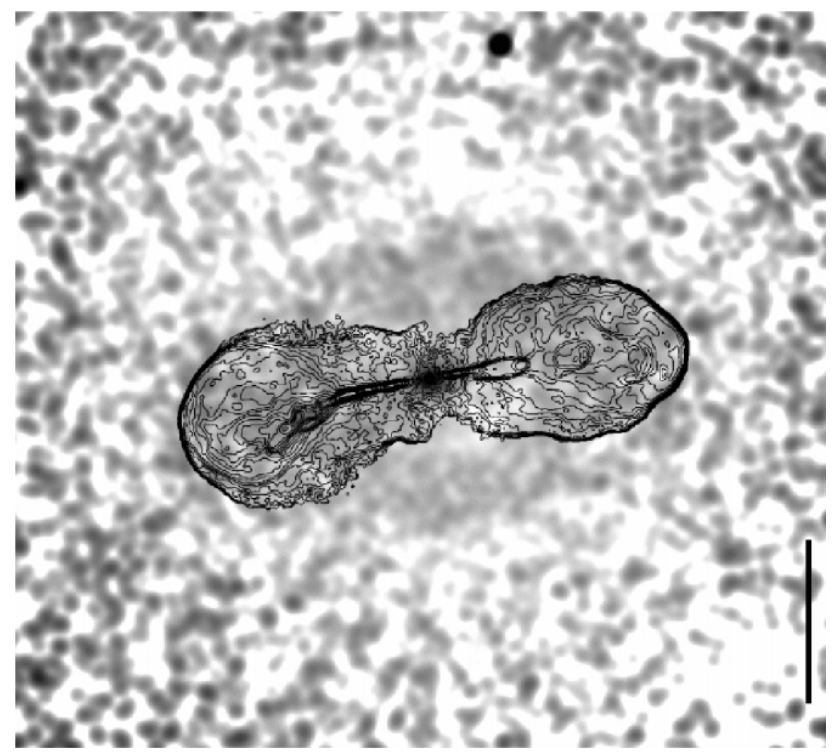

FIG. 2.-X-ray and radio images of Hercules A. The Chandra image of Fig. 1 is overlaid with $1.4 \mathrm{GHz}$ radio contours from Gizani \& Leahy (2003).

bounds this region is shown to be consistent with a shock front below.

There is an $\sim 7 \sigma$ deficit of $\mathrm{X}$-ray emission in the region $\sim 0.5$ to the southwest of the bright center in Figure $1, \sim 15^{\prime \prime}$ $(40 \mathrm{kpc})$ in radius. There is a weaker, $\sim 3 \sigma$, deficit in the Xray emission from the corresponding region to the northeast, partly masked by a bright spot of X-ray emission to the north of the center. These features resemble the cavities associated with many other cluster radio sources (e.g., McNamara et al. 2000; Fabian et al. 2000). However, they are not aligned with the axis of the radio jets and do not contain radio lobes. They might be ghost cavities (e.g., McNamara et al. 2001), but, if so, it is surprising that they lie within an active radio source.

Finally, there is a ridge of enhanced X-ray emission crossing the bright region, from $\sim 30^{\circ}$ south of east to $\sim 30^{\circ}$ north of west, roughly at right angles to the axis defined by the cavities. This feature also has no obvious association with the radio source (it forms an angle of $\sim 20^{\circ}$ with the radio jets). The excess emission appears to be thermal, due to relatively cool, dense gas, which cannot be fully supported by hydrostatic forces. The gas may be cool filaments, like those seen in other cluster central radio sources (e.g., Forman et al. 2005; Nulsen et al. 2005), or it may be a cooler disk that is partly supported by rotation.

\section{THE SHOCK FRONT IN HERCULES A}

The surface brightness profile of the bright circular region was measured in two $80^{\circ}$ sectors, approximately at right angles to the axis of the radio jet. This avoids smearing the edge in the surface brightness profile due to elongation of the bright region to the east and west. Figure 3 shows the radial surface brightness profile for the ranges of position angle (P.A.) $330^{\circ}-$ $50^{\circ}$ and $150^{\circ}-230^{\circ}$ combined. Point sources were eliminated, the background was subtracted, and the resulting profile exposure corrected. Although the data are quite noisy, there is a clear break in surface brightness at a radius of $60^{\prime \prime}(\sim 160 \mathrm{kpc})$, at the edge of the bright central region. Beyond the break, the surface brightness is well fitted by the power law, $r^{-\alpha}$ with 


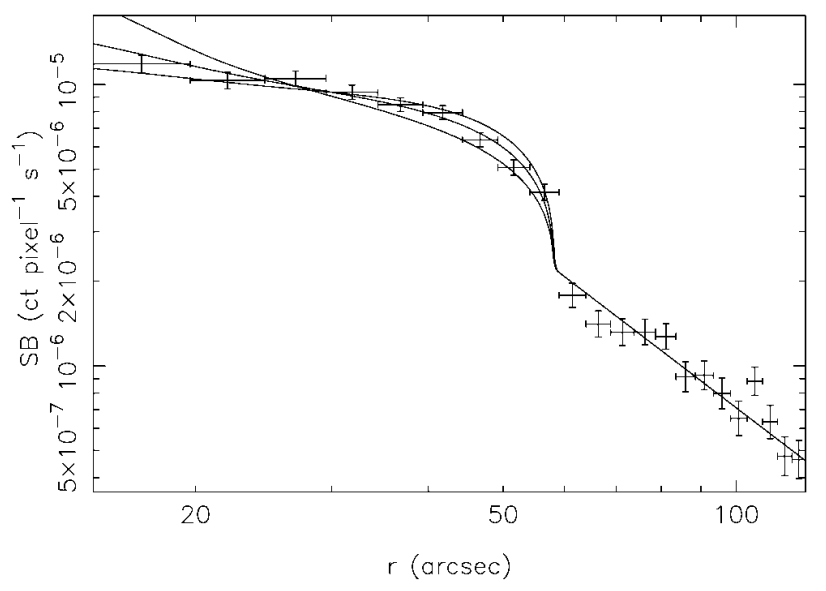

FIG. 3.-Surface brightness profile of the shock front in Hercules A. The $0.6-7.5 \mathrm{keV}$ surface brightness profile is measured in sectors from P.A. $330^{\circ}$ to $50^{\circ}$ and from P.A. $150^{\circ}$ to $230^{\circ}$, to the north and south of the AGN, at right angles to the jet axis. Surface brightness errors are $1 \sigma$ statistical errors. Radial error bars show the limits of the bins. The smooth curves are surface brightness profiles for shock models with Mach numbers of 1.51, 1.65, and 1.79, from bottom to top on the right. Models are scaled to match the observed surface brightness outside the shock.

$\alpha=2.11 \pm 0.43$ (90\%). We now consider the interpretation of this front as a shock.

To determine the strength of the shock we use a spherically symmetric, hydrodynamic model of a point explosion at the center of an initially isothermal, hydrostatic atmosphere. Before passage of the shock, the gas density is assumed to follow the power law, $\rho(r) \propto r^{-\eta}$, with $\eta=1.55$, chosen to make the surface brightness profile of the undisturbed gas match the observed profile outside the shock. The gravitational field $(g \propto 1 / r)$ is scaled to make the undisturbed atmosphere hydrostatic. The surface brightness profile is determined from the model, assuming that the temperature of the unshocked gas is $4 \mathrm{keV}$ (see below). Relative Chandra count rates in the 0.6$7.5 \mathrm{keV}$ band are computed using detector response files from near to the aim point for these observations. The XSPEC WABS $\times$ MEKAL spectral model was used, with a foreground column density of $6.4 \times 10^{20} \mathrm{~cm}^{-2}$, a redshift of 0.154 , and abundances of 0.5 times solar, appropriate for Hercules A (model surface brightness profiles are insensitive to these and the preshock temperature in the relevant temperature range). The model is self-similar, allowing it to be scaled in radius to match the location of the shock and in normalization to match observed surface brightness outside the shock.

In Figure 3 we show surface brightness profiles for model shocks with Mach numbers of 1.51, 1.65, and 1.79. A Mach 1.65 shock gives a reasonable fit to the data. Apart from the scaling, model parameters (the initial density power law, $\eta$, and preshock temperature) are constrained by observations, leaving only the Mach number of the shock free in the fit. The model has a number of shortcomings (the actual outburst is aspherical and does not inject energy in a single explosion, and the initial gas density is not a power law; Nulsen et al. 2005), so that it can only be expected to match the data over a limited range of radius behind the shock. Nevertheless, the fit provides a stringent test that this feature is due to a shock propagating into the cluster.

In order to determine the physical properties of the outburst from the model, we must determine the density and temperature

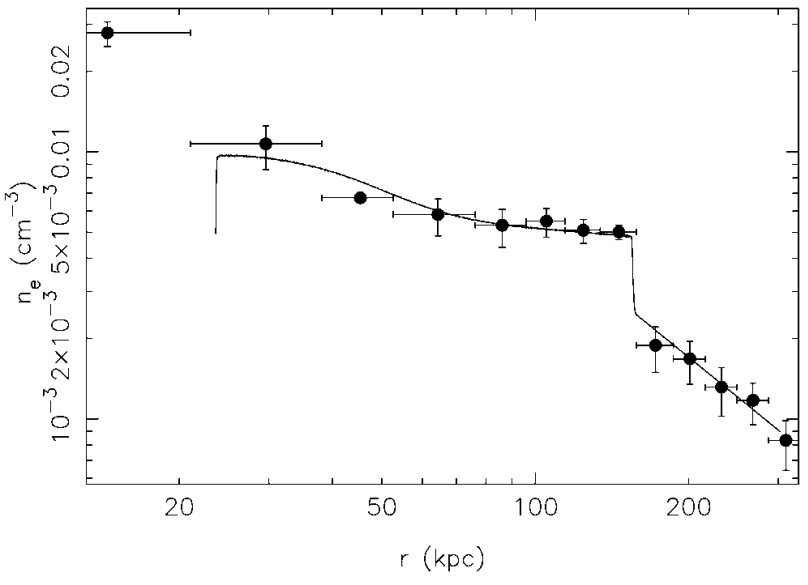

FIG. 4.-Electron density profile of the shock front in Hercules A. Deprojected electron density vs. radius in the P.A. ranges $330^{\circ}-50^{\circ}$ and $150^{\circ}-230^{\circ}$. The shock is at $158 \mathrm{kpc}$. Density error bars are $90 \%$ confidence ranges. The solid line shows the density profile for the best-fitting model.

of the unshocked gas. However, outside the shock from $1^{\prime}$ to 2.5 , in the sectors of the surface brightness profile, there are only $\sim 1250$ photons in the $0.6-7.5 \mathrm{keV}$ band. We have therefore used a single spectrum extracted from this region to determine the temperature and normalize the density of the unshocked gas. Using an absorbed MEKAL model, with $N_{\mathrm{H}}=6.4 \times$ $10^{20} \mathrm{~cm}^{-2}$, redshift $z=0.154$, and the abundance set to 0.5 , gives a temperature of $k T=3.9_{-0.6}^{+0.8} \mathrm{keV}(90 \%)$. This is consistent with previous measurements (e.g., Gizani \& Leahy 2003), suggesting that the spectrum is not significantly affected by the particle background. Assuming that the gas is spherically symmetric and its density $\rho(r) \propto r^{-1.55}$ from the shock to infinity, the normalization of the spectral fit gives an electron density of $n_{e}=1.06 \pm 0.03 \times 10^{-3} \mathrm{~cm}^{-3}$ at a radius of $276 \mathrm{kpc}(1 \cdot 72)$.

Using these parameters, the radius of the shock is $158 \mathrm{kpc}$, the time since the outburst is $t_{s}=5.9 \times 10^{7} \mathrm{yr}$, and the total energy of the outburst is $E_{s}=3 \times 10^{61}$ ergs. This energy is similar to the lobe enthalpy (Gizani \& Leahy 2004), as expected if the lobes drive the shock. The main source of uncertainty in the age of the outburst $(\sim 10 \%)$ is due to the uncertainty in the preshock temperature. The shortcomings of the model do give rise to systematic uncertainty in the shock energy, but this is unlikely to be more than a factor of $\sim 2$ (Nulsen et al. 2005).

In the temperature range $1.6-10 \mathrm{keV}$, the Chandra count rate in the band $0.6-7.5 \mathrm{keV}$ is very insensitive to gas temperature, varying $\pm 3.3 \%$ about its mean over the whole range of temperature, for a fixed emission measure. This enables us to deproject the gas density with reasonable accuracy, despite poor knowledge of the gas temperature (doubling the abundance to 1.0 would reduce the electron density by $\sim 7 \%$ ). A deprojection was done, using the method of Nulsen et al. (2005), with the gas temperature fixed at $4 \mathrm{keV}$ and other parameters as above. The resulting electron density profile is shown in Figure 4, together with the electron density profile obtained from the Mach 1.65 shock model. The results agree well with the model, clearly showing the density jump at the shock. The failure of the model for $r \lesssim 25 \mathrm{kpc}$ is a numerical artifact, but other shortcomings are expected to make the model inaccurate at small radii.

In the models, adiabatic expansion limits the size of the 
region where the temperature of the shocked gas exceeds that of the unshocked gas. Nevertheless, the strength of the shock in Hercules A makes it a good candidate for detecting the temperature rise due to the shock. For the Mach 1.65 model, after projection onto the sky, the emission measure-weighted temperature exceeds the preshock temperature by at least $20 \%$ for $110 \mathrm{kpc}<r<150 \mathrm{kpc}$. With other fit parameters as above, a spectrum extracted from this region ( $9000.6-7.5 \mathrm{keV}$ source counts) gives a temperature of $k T=6.1_{-1.2}^{+2.0} \mathrm{keV}(90 \%)$, in reasonable agreement with the model.

\section{DISCUSSION}

The mean power of the outburst in Hercules A, $P_{s}=$ $E_{s} / t_{s}=1.6 \times 10^{46} \mathrm{ergs} \mathrm{s}^{-1}$, is 2 orders of magnitude larger than the total power radiated from the region where the cooling time is shorter than $10^{10} \mathrm{yr}$. Hercules A joins a small collection of cooling flow clusters known to have large-scale shocks driven by an outburst from an AGN at the cluster center (Fabian et al. 2003; Forman et al. 2005; McNamara et al. 2005; Nulsen et al. 2005). Three of these systems, Hydra A, MS 0735.6+7421, and Hercules A, have outburst energies of $\gtrsim 10^{61} \mathrm{ergs}$. The outburst in Hercules A currently has the strongest shock, and its total energy is the second largest known (MS 0735.6+7421 is twice as energetic; McNamara et al. 2005). Along with the other systems, it has important implications for the energetics of cooling flows, the preheating of clusters, the interaction of radio sources with the ICM, and the growth of nuclear black holes (McNamara et al. 2005; Nulsen et al. 2005).

If the outburst is powered by accretion onto a black hole, then the outburst energy is $E_{s}=\epsilon M_{s} c^{2}$, where the mass $M_{s}$ was accreted to fuel the outburst and $\epsilon$ is the efficiency of jet energy production by the black hole. Unless $\epsilon>10 \%$, the mass swallowed by the black hole exceeds $1.7 \times 10^{8} M_{\odot}$ to fuel this outburst. If this mass was swallowed in a time comparable to the age of the outburst, $t_{s} \simeq 6 \times 10^{7} \mathrm{yr}$, the mass increase is hard to reconcile with a tight correlation between bulge properties and black hole mass (Gebhardt et al. 2000), unless the black hole is very massive indeed.

In our Mach 1.65 model, the shock inverts the entropy profile of gas inside $58 \mathrm{kpc}\left(22^{\prime \prime}\right)$, creating a buoyant bubble at the cluster center that would then rise. A large bubble can rise at a significant fraction of the sound speed (Churazov et al. 2001), but always more slowly than the shock front. Although the shock model is not expected to match reality closely, the cavities in Hercules A are comparable in size to the entropy inversion of the model, suggesting that this is how they were formed. This would explain the lack of radio emission from the cavities.

\section{CONCLUSIONS}

Analysis of a Chandra X-ray image of the Hercules A cluster shows that it has cavities and a shock front associated with the powerful radio source. Unusually, the cavities show no clear connection to the radio source. The shock front is elongated in the direction of the radio lobes and appears to be its cocoon shock. Fitting a simple hydrodynamic model to the surface brightness profile gives a Mach number for the shock of $\simeq 1.65$. The age of the outburst that drove the shock is $5.9 \times 10^{7} \mathrm{yr}$, and its total energy is $3 \times 10^{61}$ ergs. The deprojected density profile is consistent with the shock model and, in particular, with the density jump at the shock. Within the limits of the spectroscopic data, the temperature jump is also consistent with the shock model.

The shock outburst is highly significant for the energetics of any cooling flow in Hercules A and for the cluster as a whole. The mean mechanical power of the outburst is $\simeq 1.6 \times 10^{46}$ $\mathrm{ergs} \mathrm{s}^{-1}$, well in the range of quasar luminosities. The black hole in the AGN that drove this outburst grew by, at least, $1.7 \times$ $10^{8} M_{\odot}$ during the outburst.

We gratefully acknowledge the assistance of Alexey Vikhlinin and Maxim Markevitch in reducing the Chandra data and the referee, Martin Hardcastle, for helping to improve the Letter. P. E. J. N. was partly supported by NASA grant NAS801130. We acknowledge support from Long Term Space Astrophysics grant NAG5-11025, Chandra Archival Research grant AR2-3007X, and contract 81305-001-034V from the Department of Energy through the Los Alamos National Laboratory.

\section{REFERENCES}

Birzan, L., Rafferty, D. A., McNamara, B. R., Wise, M. W., \& Nulsen, P. E. J. 2004, ApJ, 607, 800

Carilli, C. L., Perley, R. A., \& Harris, D. E. 1994, MNRAS, 270, 173

Churazov, E., Brüggen, M., Kaiser, C. R., Böhringer, H., \& Forman, W. 2001, ApJ, 554, 261

Churazov, E., Sunyaev, R., Forman, W., \& Böhringer, H. 2002, MNRAS, 332, 729

Dreher, J. W., \& Feigelson, E. D. 1984, Nature, 308, 43

Fabian, A. C. 1994, ARA\&A, 32, 277

Fabian, A. C., et al. 2000, MNRAS, 318, L65

Fabian, A. C., Sanders, J. S., Allen, S. W., Crawford, C. S., Iwasawa, K., Johnstone, R. M., Schmidt, R. W., \& Taylor, G. B. 2003, MNRAS, 344, L43

Forman, W., et al. 2005, ApJ, in press (astro-ph/0312576)

Gebhardt, K., et al. 2000, ApJ, 539, L13

Gizani, N. A. B., \& Leahy, J. P. 2003, MNRAS, 342, 399 2004, MNRAS, 350, 865

Heinz, S., Reynolds, C. S., \& Begelman, M. C. 1998, ApJ, 501, 126
McNamara, B. R., et al. 2000, ApJ, 534, L135 2001, ApJ, 562, L149

McNamara, B. R., Nulsen, P. E. J., Wise, M. W., Rafferty, D. A., Carilli, C., Sarazin, C. L., \& Blanton, E. L. 2005, Nature, 433, 45

Motl, P. M., Burns, J. O., Loken, C., Norman, M. L., \& Bryan, G. 2004, ApJ, 606,635

Narayan, R., \& Medvedev, M. V. 2001, ApJ, 562, L129

Nulsen, P. E. J., McNamara, B. R., Wise, M. W., \& David, L. P. 2005, ApJ, in press (astro-ph/0408315)

Peterson, J. R., Kahn, S. M., Paerels, F. B. S., Kaastra, J. S., Tamura, T., Bleeker, J. A. M., Ferrigno, C., \& Jernigan, J. G. 2003, ApJ, 590, 207

Scheuer, P. A. G. 1974, MNRAS, 166, 513

Siebert, J., Kawai, N., \& Brinkmann, W. 1999, A\&A, 350, 25

Tabor, G., \& Binney, J. 1993, MNRAS, 263, 323

Trussoni, E., Feretti, L., Massaglia, S., \& Parma, P. 2001, A\&A, 366, 788

Tucker, W., \& David, L. P. 1997, ApJ, 484, 602

White, D. A., Jones, C., \& Forman, W. 1997, MNRAS, 292, 419 